\title{
Alocação de nutrientes ao longo do tronco em Pinus taeda L. aos 17 anos de idade
}

\author{
Nutrients allocation along the stem in 17-year-old Pinus taeda L.
}

Rudi Witschoreck ${ }^{\mathrm{I}}$, Mauro Valdir Schumacher ${ }^{\mathrm{II}}$

\begin{abstract}
Resumo
Este trabalho, realizado em um povoamento de Pinus taeda de 17 anos de idade no município de Cambará do Sul - RS, em Cambissolo Húmico alumínico típico, teve por objetivo estudar o padrão de alocação dos nutrientes ao longo do tronco e suas implicações para a amostragem na estimativa do estoque de nutrientes. Foram amostradas 18 árvores, distribuídas em 6 classes diamétricas, com coleta de amostras, de casca e madeira, nas seguintes alturas relativas (por cento da altura total): $\mathrm{h}_{0,1}(10 \%), \mathrm{h}_{0,3}(30 \%), \mathrm{h}_{0,5}(50 \%), \mathrm{h}_{0,7}(70 \%)$ e $\mathrm{h}_{0,9}(90 \%)$. O padrão de alocação dos nutrientes ao longo do tronco foi estudado mediante comparação de médias e ajuste de equações de regressão, correlacionando concentração de nutrientes e a altura de coleta das amostras. Testaram-se diferentes estimadores da concentração de nutrientes: teor médio aritmético (TM), teor médio ponderado pelo diâmetro da secção (TMPD) e teor médio ponderado pela biomassa da secção (TMPB); tendo este como tratamento padrão. Com exceção de Mn e Ca na madeira, todos os nutrientes apresentaram maior teor na porção superior do tronco $\left(\mathrm{h}_{0,9}\right)$, podendo ser para P 2,7 vezes superior na madeira e 5 vezes superior na casca, em relação a base do tronco $\left(\mathrm{h}_{0,1}\right)$. O elemento Ca na madeira apresentou comportamento inverso, ou seja, maior teor na base do tronco $\left(\mathrm{h}_{0,1}\right)$; enquanto $\mathrm{Mn}$ não diferiu estatisticamente entre os pontos de coleta. Entre os estimadores da concentração de nutrientes testados, o TM apresentou os maiores valores, exceto para Ca na madeira, o que acarreta superestimação do estoque de nutrientes. Para as condições do estudo, considerando o TMPB como melhor estimador da concentração de nutrientes, o intervalo de confiança para altura relativa de coleta de amostras foi estimado em $35-53 \%$ para madeira e $38-46 \%$ para casca. Palavras-chave: Ciclagem de nutriente; Nutrição florestal; Amostragem de biomassa
\end{abstract}

\begin{abstract}
This study was conducted in a 17-year-old Pinus taeda L. stand, in Cambará do Sul (RS state), in a soil classified as Humic Cambisol. It had as objective to study nutrients allocation pattern along the stem and its implications for sampling and the nutrient stock estimates. Eighteen (18) trees were sampled, distributed in 6 diametric classes, collecting bark and wood samples in the following relative heights (percentage from total height): $\mathrm{h}_{0.1}(10 \%), \mathrm{h}_{0.3}$ $(30 \%), \mathrm{h}_{0.5}(50 \%), \mathrm{h}_{0.7}(70 \%) \mathrm{e} \mathrm{h}_{0.9}(90 \%)$. The nutrient allocation pattern along the stem was studied based on the comparison of averages and regression equations, correlating nutrient content and the sampling height. Different nutrient content estimators were tested: arithmetic average content (TM), level weighted average diameter of the section (TMPD), biomass-weighted average content of the section (TMPB), being TMPB considered as the standard treatment. Excepted for $\mathrm{Mn}$ and in wood, all nutrients showed higher content in the upper part of the stem $\left(\mathrm{h}_{0.9}\right)$, sometimes 2.7 times higher in wood and 5 times higher in bark, compared to stem base $\left(\mathrm{h}_{0.1}\right)$. Calcium $(\mathrm{Ca})$ in the wood showed a reverse behavior, in other words, showed highest contents in the base of the stem $\left(\mathrm{h}_{0.1}\right)$; while Mn did not statistically differ among the sampling points. Among the tested nutrient content estimators, TM showed highest values, excepted for $\mathrm{Ca}$ in wood, that leads to nutrients stock overestimation. For the study conditions, considering TMPB as the best nutrient concentration estimator, the confidence interval for height sampling points was estimated in $35-53 \%$ for wood and $38-46 \%$ for bark.
\end{abstract}

Keywords: Nutrient cycling; Forest nutrition; Biomass sampling

\footnotetext{
${ }^{\mathrm{I}}$ Engenheiro Florestal, Dr., Pesquisador Autônomo, Laboratório de Ecologia Florestal, Centro de Ciências Rurais, Universidade Federal de Santa Maria, Av. Roraima, 1000, CEP 97105-900, Santa Maria (RS), Brasil. rwitschoreck@yahoo. com.br (ORCID: 0000-0003-3274-0815)

II Engenheiro Florestal, Dr., Professor do Departamento de Ciências Florestais, Centro de Ciências Rurais, Universidade Federal de Santa Maria, Av. Roraima, 1000, CEP 97105-900, Santa Maria (RS), Brasil. schumacher@pesquisador.cnpq.br (ORCID: 0000-0003-3277-5671)
} 


\section{Introdução}

O conhecimento da distribuição de biomassa e dos nutrientes nos diferentes compartimentos e fases de desenvolvimento dos povoamentos florestais é indispensável no estudo da ciclagem de nutrientes e na definição de inúmeras práticas de manejo, principalmente as que visam à manutenção da capacidade produtividade, tais como: seleção de material genético, idade de colheita, intensidade de remoção de biomassa, manejo de resíduos florestais, adubação de reposição, entre outras (LANDSBERG, 1986; REIS; BARROS, 1990). A despeito da importância desses estudos, as metodologias de coleta de amostras para a estimativa do estoque de nutrientes em espécies florestais ainda são divergentes. Em geral, reconhece-se a grande variação na concentração de nutrientes entre os componentes das árvores, e por essa razão, além das vantagens operacionais, nos procedimentos de campo, a biomassa é triada e amostrada nos diferentes componentes: folhas, galhos, casca, madeira, raízes, entre outros. No entanto, as variações intracomponentes, geralmente, não são consideradas nos procedimentos amostrais, o que é especialmente importante para o tronco, que é o principal compartimento envolvido na exportação de nutrientes por meio da colheita florestal, e que em povoamentos adultos pode representar mais de $80 \%$ da biomassa acima do solo.

O tronco, principalmente madeira em povoamentos adultos, pode não ser o componente com as maiores concentrações de nutrientes, mas em função do grande acúmulo de biomassa, a coleta de amostras não representativas pode provocar desvios significativos nas estimativas do estoque de nutrientes.

Se a variação na concentração de nutrientes for tão expressiva como alguns estudos indicam, secções diferentes do tronco, casca e madeira, teriam que ser tratadas praticamente como componentes diferentes por ocasião da amostragem de biomassa, exigindo coleta de mais de uma amostra ao longo do tronco (WITSCHORECK; SCHUMACHER, 2013).

Devido à grande importância do tronco, como órgão de acúmulo de biomassa e nutrientes, vários trabalhos têm sido desenvolvidos para esclarecer os padrões de distribuição dos nutrientes e definir procedimentos de coleta e estimativa do estoque de nutrientes na casca e na madeira do tronco (ATTIWILL, 1980; ZEN; POGGIANI; COUTO, 1981; ANDRAE, 1983; ANDRAE; KRAPFENBAUER, 1983a; 1983b; FREITAS, 2000; HOPPE, 2003; SILVA, 1996 apud BELLOTE; SILVA, 2004; RUBILAR; ALLEN; KELTING, 2005; SAIDELLES, 2005; MORO et al., 2007; WITSCHORECK, 2008; WITSCHORECK; SCHUMACHER, 2013).

O objetivo geral do trabalho foi estudar a alocação de nutrientes na casca e na madeira do tronco das árvores de Pinus taeda, e suas implicações na amostragem de biomassa e estimativa do estoque de nutrientes. Para isso, as seguintes hipóteses foram testadas: H1. Existe diferença significativa no teor de nutrientes entre amostras de casca e madeira coletadas em diferentes posições ao longo do tronco. H2. A média aritmética de amostras coletadas em diferentes pontos ao logo tronco é representativa da concentração de nutrientes, para fins de estimativa do estoque de nutrientes. H3. O estoque de nutrientes do tronco pode ser estimado adequadamente com a coleta de uma única amostra de casca e de madeira.

\section{Material e método}

O estudo foi realizado em um povoamento de Pinus taeda da empresa Cambará S.A., no município de Cambará do Sul, região fisiográfica dos Campos de Cima da Serra, nordeste do Estado do Rio Grande do Sul, localizado na latitude $29^{\circ} 00^{\prime} 07^{\prime}$ ' sul, longitude $50^{\circ} 04^{\prime} 57^{\prime \prime}$ oeste e altitude de $1.057 \mathrm{~m}$.

O clima da região é o temperado, Cfb segundo a classificação de Köppen, com temperatura média anual de $15,5^{\circ} \mathrm{C}$, sendo $20,9^{\circ} \mathrm{C}$ para as máximas, $10,9^{\circ} \mathrm{C}$ para as mínimas, um extremo para as mínimas absolutas de $-7,2^{\circ} \mathrm{C}$, observados na estação meteorológica de Cambará do Sul, no período de 1997-2007 (INSTITUTO NACIONAL DE METEOROLOGIA, 2008). Para o mesmo período, foi registrada uma precipitação média de $159 \mathrm{~mm}$ mensais ou $1.906 \mathrm{~mm}$ anuais. 
O solo é um Cambissolo Húmico alumínico típico, pertencente à unidade de mapeamento Bom Jesus, que tem como principais características o elevado acúmulo de matéria orgânica no horizonte superficial, baixa saturação de bases, alta acidez, teores trocáveis de $\mathrm{Al} \geq 4 \mathrm{cmol} / \mathrm{dm}^{3}$ e saturação de $\mathrm{Al} \geq$ $50 \%$ (STRECK et al., 2008). Na Tabela 1 é possível verificar as características do solo da área de estudo antes da coleta de dados, proveniente da coleta de 4 amostras compostas em cada profundidade.

Tabela 1- Caracterização do solo, sob plantio de Pinus taeda aos 17 anos de idade, em Cambará do Sul - RS.

Table 1 - Soil characterization, under a 17-year-old Pinus taeda stand, in Cambará do Sul - RS state.

\begin{tabular}{|c|c|c|c|c|c|c|c|c|c|c|}
\hline \multirow{2}{*}{ Prof. (cm) } & Argila & M.O. & \multirow{2}{*}{$\begin{array}{c}\mathrm{pH} \\
\left(\mathrm{H}_{2} \mathrm{O}\right)\end{array}$} & $\mathbf{P}$ & $\mathbf{K}$ & $\mathrm{Ca}$ & Mg & Al & CTC ${ }_{\text {efetiva }}$ & \multirow{2}{*}{$\frac{V}{(\%)}$} \\
\hline & \multicolumn{2}{|c|}{$\left(\mathrm{g} \mathrm{kg}^{-1}\right)$} & & \multicolumn{2}{|c|}{$\mathrm{mg} \mathrm{dm}^{-3}$} & \multicolumn{4}{|c|}{$\mathrm{cmol}_{\mathrm{c}} \mathrm{dm}^{-3}$} & \\
\hline $0-20$ & 410 & 55,8 & 4,3 & 2,6 & 63,3 & 3,6 & 1,2 & 5,4 & 11,1 & 25 \\
\hline $20-40$ & 516 & 53,4 & 4,3 & 1,7 & 31,0 & 1,2 & 0,3 & 7,0 & 8,6 & 10 \\
\hline $40-60$ & 531 & 28,1 & 4,4 & 1,0 & 17,5 & 0,9 & 0,2 & 6,6 & 7,4 & 7 \\
\hline
\end{tabular}

Em que: Argila pelo método do densímetro; matéria orgânica (M.O.), digestão por combustão úmida $\left(\mathrm{K}_{2} \mathrm{Cr}_{2} \mathrm{O}_{7}+\mathrm{H}_{2} \mathrm{SO}_{4}\right) ; \mathrm{P}$ disponível e K trocável, extração do solo com solução Mehlich - 1; Ca, Mg e Al trocáveis, extração por solução de $\mathrm{KCl}$ (1 mol $\left.\mathrm{L}^{-1}\right) ; \mathrm{V}=$ saturação por bases.

A coleta de biomassa foi baseada na amplitude diamétrica $(18,1 \mathrm{~cm}-50,8 \mathrm{~cm}$ de DAP), com amostragem de 18 árvores, distribuídas em 6 classes de diâmetro (três árvores por classe), com intervalo entre classes de 5,5 cm. Após o abate das árvores-amostra, foram retirados discos do tronco e desses amostras representativas de madeira e casca, com aproximadamente 100 gramas, nas seguintes alturas relativas: $h 0,1$; $h 0,3 ; h 0,5 ; h 0,7$ e $h 0,9$, respectivamente, $10 \%, 30 \%, 50 \%, 70 \%$ e $90 \%$ da altura total (Figura 1).

A biomassa seca total de casca e madeira do tronco $(B)$ foi obtida pelo somatório dos valores individuais dos segmentos $b 1, b 2, b 3, b 4$ e $b 5$, equivalentes, respectivamente, as secções: 0 - 20, 20 - 40, $40-60,60-80$ e $80-100 \%$ da altura total das árvores-amostra (Figura 1).

Figura 1 - Desenho esquemático da coleta de amostras, de casca e madeira, ao longo do tronco das árvores-amostra de Pinus taeda, aos 17 anos de idade, em Cambará do Sul - RS.

Figure 1 - Wood and bark collecting samples (schematic draw) along the stem in 17-year-old Pinus taeda sampled trees, in Cambará do Sul - RS state.

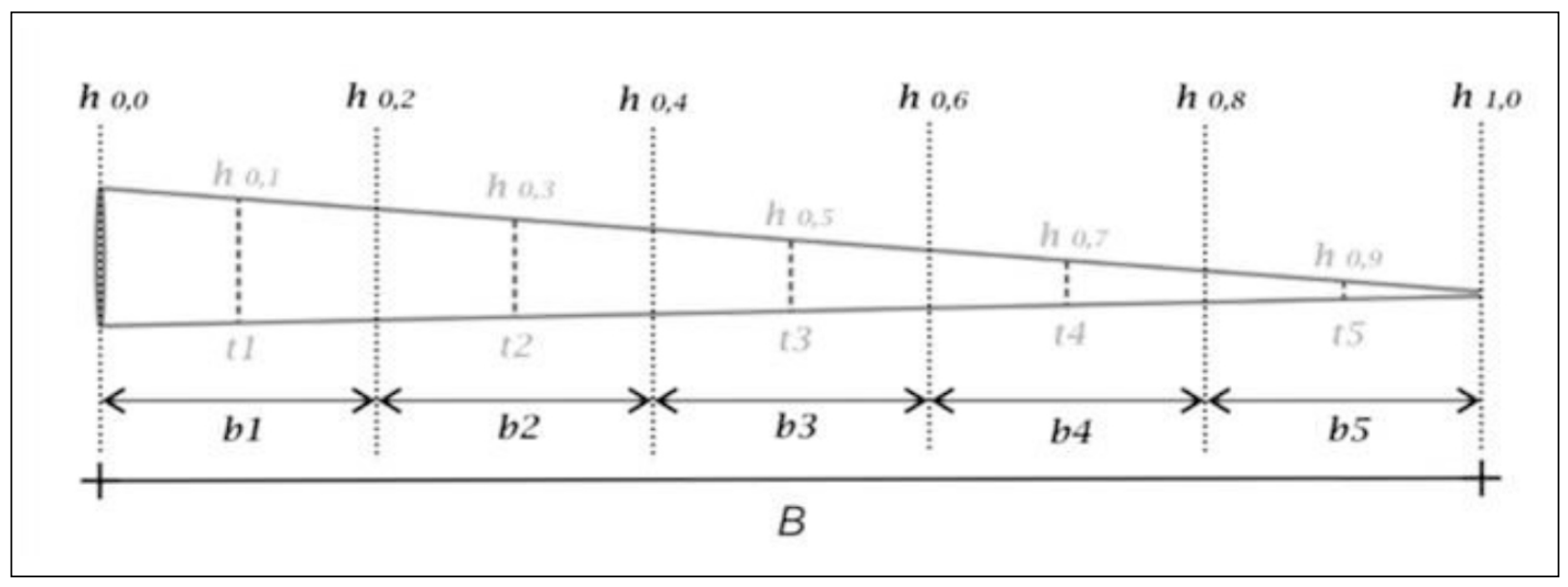


Para avaliar diferentes estimadores da concentração de nutrientes, para determinar o estoque de nutrientes na casca e na madeira do tronco, as informações referentes à biomassa e análises químicas receberam diferentes abordagens. Partiu-se do princípio que a estimativa mais precisa do estoque de nutrientes é obtida mediante a coleta de várias amostras $(h 0,1 ; h 0,3 ; h 0,5 ; h 0,7$ e $h 0,9)$, de casca e madeira, ao longo do tronco (Figura 1). Para a situação considerada ideal, a biomassa de cada segmento ( $b 1, b 2, b 3, b 4$ e $b 5)$ foi considerada individualmente, o teor ( $t i)$ obtido em cada secção foi ponderado em relação à biomassa $(b i)$, obtendo-se o teor médio ponderado em relação à biomassa (TMPB). Com o objetivo de facilitar o processo amostral, devido à dificuldade de coleta e determinação da biomassa (e aproveitando a grande correlação do diâmetro da secção do tronco e a respectiva biomassa), o segundo estimador considerado foi o teor ponderado em relação ao diâmetro (TMPD); além da média aritmética dos teores $t 1, t 2, t 3, t 4$ e $t 5$ (TM). Na comparação de médias entre os estimadores da concentração de nutrientes (TMPB, TMPD e TM), foi utilizado o teste de Tukey a $5 \%$ de probabilidade de erro.

No Laboratório de Ecologia Florestal, do Departamento de Ciências Florestais da Universidade Federal de Santa Maria, as amostras coletadas foram secas em estufa com circulação e renovação de ar, a temperatura de $65^{\circ} \mathrm{C}$, até estabilização do peso, para determinação do teor de umidade. Após a secagem, as amostras foram moídas em moinho de lâminas do tipo Wiley com peneira de 20 mesh. As determinações analíticas foram realizadas de acordo com a metodologia de Miyazawa et al. (1999). O nitrogênio foi determinado pelo método Kjeldhal, no extrato de digestão sulfúrica; boro por espectrofotometria com digestão seca; e os demais elementos no extrato de digestão nítrico-perclórica, sendo cálcio, magnésio, cobre, ferro, manganês e zinco por espectrofotometria de absorção atômica, fósforo por espectrofotometria (VIS), potássio por fotometria de chama e enxofre por turbidimetria.

Além da comparação de médias, pelo teste de Tukey a 5\% de probabilidade de erro, dos teores de nutrientes nos diferentes pontos de coleta de amostras $(h i)$, a variabilidade na distribuição dos nutrientes nas diferentes posições ao longo do tronco foi estudada por meio do ajuste de equações de regressão. As equações de regressão foram ajustadas tendo como variável de saída o teor de nutrientes, sendo $\mathrm{N}$, $\mathrm{P}, \mathrm{K}, \mathrm{Ca}, \mathrm{Mg}$ e S, em $\mathrm{g} \mathrm{kg}^{-1} \mathrm{e} \mathrm{B}, \mathrm{Cu}, \mathrm{Fe}, \mathrm{Mn}$ e $\mathrm{Zn}$ em $\mathrm{mg} \mathrm{kg}^{-1}$ ), e como variável preditiva a altura de coleta de amostras, 0,$1 ; 0,3 ; 0,5 ; 0,7$ e 0,9 , respectivamente, $h 0,1 ; h 0,3 ; h 0,5 ; h 0,7$ e $h 0,9$. A seleção das equações, de melhor ajuste aos dados, teve como base as estatísticas: coeficiente de determinação $\left(\mathrm{R}^{2}\right)$, erro-padrão da estimativa, significância da estatística $\mathrm{F}$ da análise de variância e a significância estatística dos coeficientes das equações. Com base nas equações de regressão selecionadas para cada nutriente, foi determinado o intervalo de confiança, a 5\% de probabilidade de erro, para a altura de coleta de amostras de casca e de madeira do tronco.

\section{Resultados e discussão}

Na Tabela 2, excetuando-se o Mn na madeira, todos os nutrientes, em ambos os componentes, casca e madeira, apresentaram diferença estatística no teor nutricional entre os pontos de coleta de amostras ao longo do tronco, em pelo menos um contraste. Com exceção do Mn (invariável) e do Ca na madeira, todos os nutrientes apresentaram maior teor na extremidade superior do tronco: ponto de amostragem $\mathrm{h}_{0,9}$; podendo ser para o P 2,7 vezes superior na madeira e 5 vezes superior na casca, em relação ao ponto $h_{0,1}$ (Tabela 2 ). 
Tabela 2 - Teores médios de nutrientes, na casca e na madeira, em diferentes pontos ao longo do tronco, em um povoamento de Pinus taeda, com 17 anos de idade, em Cambará do Sul - RS.

Table 2 - Average nutrient contents, in wood and bark, in different points along the stem, in a 17-yearold Pinus taeda stand, in Cambará do Sul - RS state.

\begin{tabular}{|c|c|c|c|c|c|}
\hline \multirow{2}{*}{ Nutrientes } & \multicolumn{5}{|c|}{ Pontos de amostragem (altura relativa) } \\
\hline & $\mathbf{h}_{0,1}$ & $\mathbf{h}_{0,3}$ & $\mathbf{h}_{0,5}$ & $\mathbf{h}_{0,7}$ & $\mathbf{h}_{0,9}$ \\
\hline \multicolumn{6}{|c|}{ Madeira do tronco } \\
\hline & 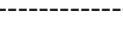 & - & - & 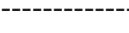 & \\
\hline $\mathrm{N}$ & $1,20 \mathrm{c}$ & $1,30 \mathrm{bc}$ & $1,31 \mathrm{bc}$ & $1,46 b$ & $2,10 \mathrm{a}$ \\
\hline $\mathrm{P}$ & $0,06 \mathrm{c}$ & $0,07 \mathrm{~cd}$ & $0,08 \mathrm{bc}$ & $0,10 \mathrm{~b}$ & $0,16 \mathrm{a}$ \\
\hline K & $0,53 \mathrm{c}$ & $0,56 \mathrm{bc}$ & $0,59 \mathrm{bc}$ & $0,66 b$ & $0,95 \mathrm{a}$ \\
\hline $\mathrm{Ca}$ & $0,75 \mathrm{a}$ & $0,68 \mathrm{ab}$ & $0,68 \mathrm{ab}$ & $0,67 \mathrm{ab}$ & $0,60 \mathrm{~b}$ \\
\hline $\mathrm{Mg}$ & $0,27 b$ & $0,26 \mathrm{~b}$ & $0,28 b$ & $0,28 b$ & $0,35 \mathrm{a}$ \\
\hline $\mathrm{S}$ & $0,36 \mathrm{~b}$ & $0,33 b$ & $0,33 b$ & $0,36 \mathrm{~b}$ & $0,44 \mathrm{a}$ \\
\hline $\mathrm{B}$ & $4,20 \mathrm{c}$ & $4,47 \mathrm{bc}$ & $4,61 b c$ & $5,31 \mathrm{~b}$ & $6,83 \mathrm{a}$ \\
\hline $\mathrm{Cu}$ & $1,26 \mathrm{c}$ & $1,40 \mathrm{c}$ & $1,51 \mathrm{c}$ & $1,90 \mathrm{~b}$ & $2,82 \mathrm{a}$ \\
\hline $\mathrm{Fe}$ & $13,71 b$ & $12,49 b$ & $11,45 b$ & $12,49 b$ & $17,62 \mathrm{a}$ \\
\hline $\mathrm{Mn}$ & $35,31 \mathrm{a}$ & $32,10 \mathrm{a}$ & $34,75 a$ & $32,95 \mathrm{a}$ & $36,87 \mathrm{a}$ \\
\hline $\mathrm{Zn}$ & $3,30 \mathrm{c}$ & $3,25 \mathrm{c}$ & $3,35 c$ & $4,31 \mathrm{~b}$ & $8,56 \mathrm{a}$ \\
\hline \multicolumn{6}{|c|}{ Casca do tronco } \\
\hline $\mathrm{N}$ & $2,60 \mathrm{e}$ & $2,95 \mathrm{~d}$ & $3,40 \mathrm{c}$ & $4,37 \mathrm{~b}$ & $6,57 \mathrm{a}$ \\
\hline $\mathrm{P}$ & $0,22 \mathrm{~d}$ & $0,26 \mathrm{~d}$ & $0,35 \mathrm{c}$ & $0,50 \mathrm{~b}$ & $0,81 \mathrm{a}$ \\
\hline K & $0,70 \mathrm{e}$ & $0,88 \mathrm{~d}$ & $1,35 \mathrm{c}$ & $2,11 b$ & $3,53 \mathrm{a}$ \\
\hline $\mathrm{Ca}$ & $1,45 \mathrm{a}$ & $0,97 \mathrm{~b}$ & $0,93 b$ & $1,05 \mathrm{~b}$ & $1,54 \mathrm{a}$ \\
\hline $\mathrm{Mg}$ & $0,42 d$ & $0,47 d$ & $0,60 \mathrm{c}$ & $0,78 \mathrm{~b}$ & $1,27 \mathrm{a}$ \\
\hline $\mathrm{S}$ & $0,36 \mathrm{~d}$ & $0,37 \mathrm{~cd}$ & $0,41 \mathrm{c}$ & $0,47 b$ & $0,64 \mathrm{a}$ \\
\hline $\mathrm{B}$ & $9,60 \mathrm{c}$ & $10,33 c$ & $10,78 b c$ & $12,65 b$ & $16,81 \mathrm{a}$ \\
\hline $\mathrm{Cu}$ & $4,49 b$ & $4,57 \mathrm{~b}$ & $4,59 b$ & $4,88 b$ & $5,72 \mathrm{a}$ \\
\hline $\mathrm{Fe}$ & $172,96 \mathrm{c}$ & $199,00 \mathrm{~b}$ & $196,14 b$ & $209,41 b$ & $267,00 \mathrm{a}$ \\
\hline $\mathrm{Mn}$ & $29,3 \mathrm{~d}$ & $35,30 \mathrm{c}$ & $38,61 \mathrm{c}$ & $48,32 b$ & $68,46 \mathrm{a}$ \\
\hline $\mathrm{Zn}$ & $7,32 \mathrm{e}$ & $11,51 \mathrm{~d}$ & $15,54 \mathrm{c}$ & $24,96 b$ & $35,50 \mathrm{a}$ \\
\hline
\end{tabular}

Em que: Médias seguidas pela mesma letra na horizontal não diferem estatisticamente pelo teste de Tukey em nível de 5\% de probabilidade de erro.

A biomassa relativa também apresentou grande diferença entre as secções do tronco. Devido à conicidade do tronco, o segmento de $0-20$, na base, representa $42,8 \%$ da biomassa total, tanto para casca como para madeira, ao passo que, o segmento de 80 - 100, na parte superior do tronco, acumula somente $2,3 \%$ da casca e apenas $1,2 \%$ da madeira (Figura 2). 
Figura 2 - Biomassa relativa de casca e madeira, em diferentes secções ao longo do tronco, em um povoamento de Pinus taeda, com 17 anos de idade, em Cambará do Sul - RS.

Figure 2 - Wood and bark relative biomass, in different sections along the stem, in a 17-year-old Pinus taeda stand, in Cambará do Sul - RS state.

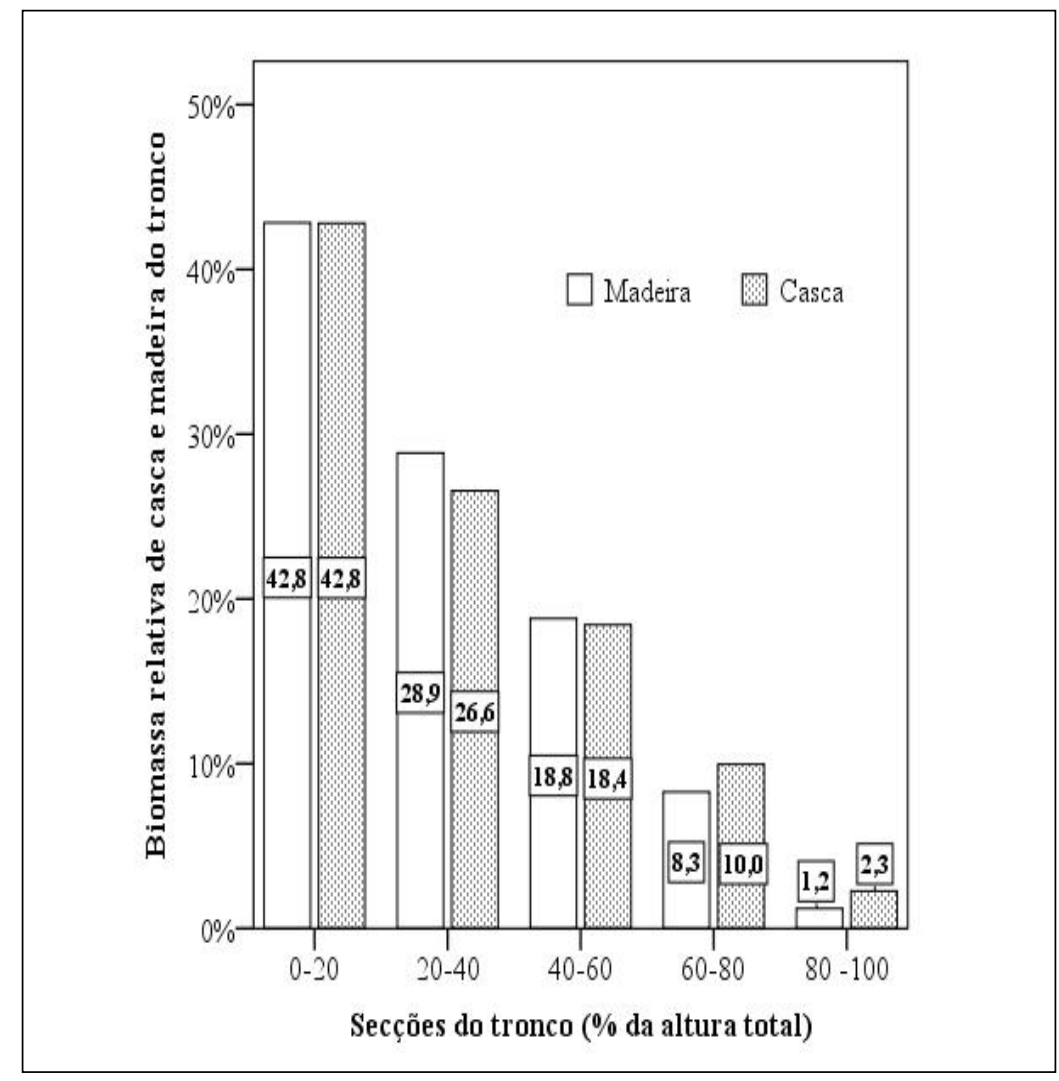

O padrão inverso entre a distribuição de biomassa e dos nutrientes, respectivamente, decrescente e crescente (para a maioria dos nutrientes), da base em direção ao ápice do tronco, sugere que o tronco não deve ser considerado como uma unidade em termos de alocação de nutrientes, o que exige uma abordagem mais criteriosa na coleta de amostras representativas de casca e madeira para estimar adequadamente o estoque de nutrientes. Nessas condições, a média aritmética não é um bom estimador da concentração de nutrientes, provocando superestimação do estoque de nutrientes, para a maioria, em ambos os componentes, mas principalmente na casca, em que a variação da concentração ao longo do tronco é maior. No caso da casca e da madeira, como a média aritmética atribui pesos iguais a cada subamostra, ela só pode ser utilizada na estimativa do estoque de nutrientes quando o tronco apresenta forma cilíndrica, de modo que cada secção apresente uma quantidade equivalente de biomassa, ou na ausência de diferença estatística da concentração de nutrientes entre os pontos de coleta de amostras ao longo do tronco.

Na Tabela 3 apresenta-se a comparação de médias entre os diferentes estimadores da concentração de nutrientes na casca e na madeira do tronco de Pinus taeda, onde prevalece os maiores valores para TM. Com exceção de $\mathrm{Ca}$ e $\mathrm{Cu}$ na casca, e Ca, Mg, S, Fe e Mn na madeira, TM difere estatisticamente de TMPB. Em virtude do acúmulo de biomassa na base do tronco (Figura 2) e a prevalência dos teores mais elevados na extremidade superior do tronco (com menor representatividade em alocação de biomassa), a média aritmética (TM), superestima a concentração de nutrientes e consequentemente o estoque de nutrientes na casca e na madeira do tronco. A superestimativa do teor nutricional, com base no TM, pode ser de $59,8 \%$ para o $\mathrm{K}$ na casca e de até $32,9 \%$ para $\mathrm{Zn}$ na madeira, quando comparado com o TMPB (Figura 3). Considerando-se que neste estudo a biomassa de tronco de Pinus taeda corresponde a $80,8 \%$ do total acima do solo, sendo $8,6 \%$ de casca e $72,2 \%$ de madeira (WITSCHORECK, 2008), os erros na estimativa do estoque total de nutrientes com o uso do TM podem ser expressivos (Figura 3). 
Tabela 3 - Comparação estatística do teor médio (TM), teor médio ponderado pelo diâmetro da secção (TMPD) e teor médio ponderado pela biomassa da secção (TMPB), na casca e na madeira do tronco, em um povoamento de Pinus taeda, com 17 anos de idade, em Cambará do Sul - RS.

Table 3 - Statistical comparison of arithmetic average content (TM), level weighted average diameter of the section (TMPD) and biomass-weighted average content of the section (TMPB), in a 17-year-old Pinus taeda stand, in Cambará do Sul - RS state.

\begin{tabular}{|c|c|c|c|}
\hline \multirow{2}{*}{ Nutrientes } & \multicolumn{3}{|c|}{ Estimadores da concentração de nutrientes } \\
\hline & TMPB & TMPD & TM \\
\hline \multicolumn{4}{|c|}{ Casca do tronco } \\
\hline \multicolumn{4}{|c|}{ - } \\
\hline $\mathrm{N}$ & $3,10 \mathrm{~b}$ & $3,30 \mathrm{~b}$ & $3,98 \mathrm{a}$ \\
\hline $\mathrm{P}$ & $0,30 \mathrm{~b}$ & $0,33 b$ & $0,43 \mathrm{a}$ \\
\hline $\mathrm{K}$ & $1,07 \mathrm{c}$ & $1,22 \mathrm{~b}$ & $1,71 \mathrm{a}$ \\
\hline $\mathrm{Ca}$ & $1,18 \mathrm{a}$ & $1,15 \mathrm{a}$ & $1,19 \mathrm{a}$ \\
\hline $\mathrm{Mg}$ & $0,52 b$ & $0,56 \mathrm{~b}$ & $0,71 \mathrm{a}$ \\
\hline $\mathrm{S}$ & $0,39 \mathrm{~b}$ & $0,40 \mathrm{~b}$ & $0,45 \mathrm{a}$ \\
\hline \multicolumn{4}{|c|}{ - } \\
\hline B & $10,44 \mathrm{~b}$ & $10,80 \mathrm{ab}$ & $12,03 \mathrm{a}$ \\
\hline $\mathrm{Cu}$ & 4,60a & $4,65 \mathrm{a}$ & $4,85 \mathrm{a}$ \\
\hline $\mathrm{Fe}$ & $189,56 \mathrm{~b}$ & $194,53 b$ & $208,90 \mathrm{a}$ \\
\hline $\mathrm{Mn}$ & $35,33 b$ & $37,52 b$ & $44,00 \mathrm{a}$ \\
\hline $\mathrm{Zn}$ & $12,39 \mathrm{c}$ & $14,14 \mathrm{~b}$ & $18,97 \mathrm{a}$ \\
\hline \multicolumn{4}{|c|}{ Madeira do tronco } \\
\hline & ----- & ----- & ---- \\
\hline $\mathrm{N}$ & $1,28 \mathrm{~b}$ & $1,33 \mathrm{~b}$ & $1,47 \mathrm{a}$ \\
\hline $\mathrm{P}$ & $0,08 \mathrm{~b}$ & $0,08 \mathrm{~b}$ & $0,10 \mathrm{a}$ \\
\hline $\mathrm{K}$ & $0,56 \mathrm{~b}$ & $0,59 \mathrm{~b}$ & $0,66 \mathrm{a}$ \\
\hline $\mathrm{Ca}$ & $0,71 \mathrm{a}$ & $0,70 \mathrm{a}$ & $0,68 \mathrm{a}$ \\
\hline $\mathrm{Mg}$ & $0,27 \mathrm{a}$ & $0,28 \mathrm{a}$ & $0,29 \mathrm{a}$ \\
\hline $\mathrm{S}$ & $0,35 \mathrm{a}$ & $0,35 \mathrm{a}$ & $0,36 \mathrm{a}$ \\
\hline \multicolumn{4}{|c|}{ - } \\
\hline B & $4,48 b$ & $4,65 \mathrm{ab}$ & $5,08 \mathrm{a}$ \\
\hline $\mathrm{Cu}$ & $1,42 b$ & $1,52 \mathrm{~b}$ & $1,78 \mathrm{a}$ \\
\hline $\mathrm{Fe}$ & $12,87 \mathrm{a}$ & $12,87 \mathrm{a}$ & $13,55 \mathrm{a}$ \\
\hline $\mathrm{Mn}$ & $34,10 \mathrm{a}$ & $34,04 \mathrm{a}$ & $34,40 \mathrm{a}$ \\
\hline $\mathrm{Zn}$ & $3,43 b$ & $3,68 \mathrm{~b}$ & $4,56 \mathrm{a}$ \\
\hline
\end{tabular}

Em que: Médias seguidas pela mesma letra na horizontal não diferem estatisticamente pelo teste de Tukey em nível de $5 \%$ de probabilidade de erro.

O TMPD também apresentou tendência à superestimação da concentração de nutrientes em relação ao TMPB, no entanto, a diferença foi estatisticamente significativa apenas para $\mathrm{K}$ e Zn na casca. Os maiores desvios do TMPD em relação ao TMPB foram de $14,1 \%$ na casca e $7,3 \%$ na madeira, ambos para o elemento Zn. O TMPD se mostra uma alternativa bem mais precisa que o TM e apresenta vantagens 
por ocasião da amostragem, em relação ao TMPB, já que não é necessário determinar a biomassa de casca e madeira por secção, apenas a biomassa total de cada um desses componentes e o diâmetro nos pontos de coleta de amostras. Ou ainda, no caso da obtenção apenas de um teor representativo, mede-se apenas o diâmetro nos pontos de coleta das amostras.

Figura 3 -Variação relativa (\%) do teor médio (TM) e do teor médio ponderado pelo diâmetro da secção (TMPD) em relação ao teor médio ponderado pela biomassa (TMPB), na casca e na madeira do tronco, em um povoamento de Pinus taeda, com 17 anos de idade, em Cambará do Sul - RS.

Figure 3 - Relative variation of arithmetic average content (TM) and of the average level measured by the diameter of the section (TMPD) in relation to the biomass-weighted average (TMPB), in wood and bark stem, in a 17-year-old Pinus taeda stand, in Cambará do Sul - RS state.

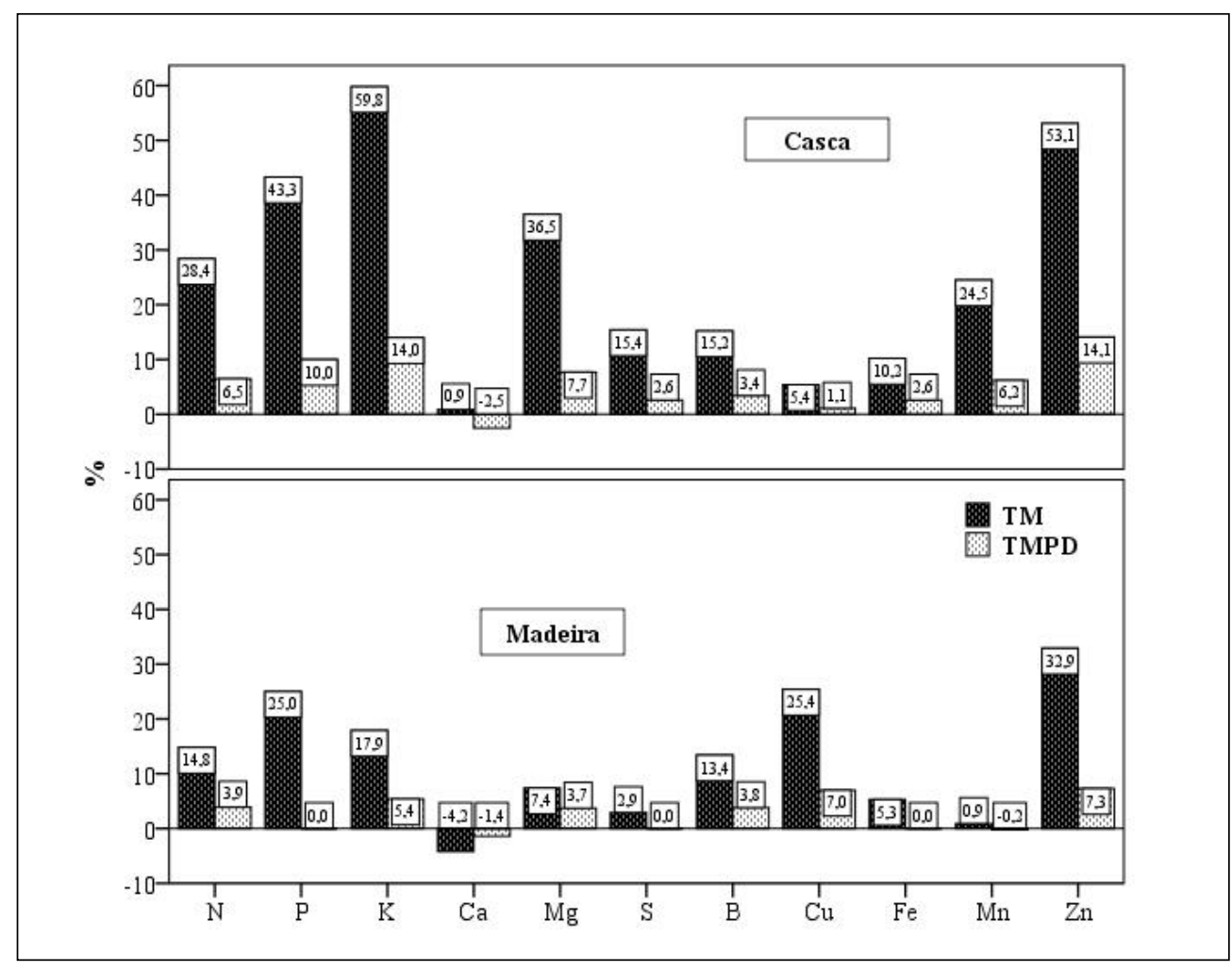

Com base no que foi apresentado, depreende-se que o número de amostras coletadas ao longo do tronco não garante boas estimativas do estoque de nutrientes, dependendo do método de amostragem. As maiores distorções foram provocadas por amostras coletadas na porção terminal superior do tronco, a qual apresenta baixa representatividade em termos de acúmulo de biomassa e concentrações de nutrientes muito distintas em relação ao restante do tronco. Deste modo, na avaliação do estoque de nutrientes no tronco, o processo de estimativa deve considerar não só a concentração de nutrientes, mas a representatividade em termos de acúmulo de biomassa de cada subamostra de casca e madeira. Da mesma forma, devido à variabilidade nutricional, é possível concluir que na estimativa do conteúdo de nutrientes de porções específicas do tronco não deve ser utilizado resultados analíticos de amostras coletadas em outras posições ao longo do tronco, sobretudo nas extremidades (ZEN; POGGIANI; COUTO, 1981).

A variação dos teores de nutrientes nas diferentes posições ao longo do tronco está ligada, principalmente, à diferença de idade dos tecidos e à mobilidade dos nutrientes (ATTIWILL, 1980; ANDRAE, 1983; ANDRAE; KRAPFENBAUER, 1983a; 1983b; RUBILAR; ALLEN; KELTING, 2005; WITSCHORECK; SCHUMACHER, 2013). A diferenciação na concentração de nutrientes, entre componentes e dentro de um mesmo componente, tende a se intensificar com a idade, em decorrência da ciclagem interna (ciclagem bioquímica), que desloca nutrientes de tecidos senescentes para regiões com 
maior atividade metabólica (REIS; BARROS, 1990; PALLARDY, 2008); o que é especialmente importante no tronco, por meio da transformação de alburno em cerne. Segundo Burger e Richter (1991), o alburno, localizado nas camadas mais periféricas do tronco, constitui-se em tecido mais ativo fisiologicamente que o cerne (parte central do tronco) e diferentemente do cerne, o alburno é constituído por células parenquimáticas vivas que encerram substâncias nutritivas como amido, açúcares, proteínas etc.; o que lhe atribui maior concentração de nutrientes. Da mesma forma, considerando-se que o crescimento das árvores ocorre mediante a sobreposição de camadas de lenho, resultante da atividade dos tecidos meristemáticos, decorrente dos sucessivos ciclos de crescimento ao longo do tempo (BURGER; RICHTER, 1991; PALLARDY, 2008), a idade média dos tecidos lenhosos e a proporção de cerne varia em ordem decrescente no sentido longitudinal, da base da árvore em direção ao ápice e no sentido radial, da medula em direção à periferia do tronco. Deste modo, enquanto os elementos imóveis ou pouco móveis acumulam-se na base do tronco ou não variam expressivamente ao longo do tronco, os nutrientes com alta mobilidade apresentam os maiores teores na extremidade superior do tronco (ZEN; POGGIANI; COUTO, 1981; ANDRAE; KRAPFENBAUER, 1983a; PEREIRA et al., 1984; REIS; BARROS, 1990; HOPPE, 2003). Segundo Waring e Schlesinger (1985), a proeminência do estoque de substâncias de reserva na base do tronco e raízes grossas é consistente para muitas espécies arbóreas, o que pode explicar a elevação dos teores de alguns nutrientes na parte inferior do tronco. Attiwill (1980) quando estudou a ciclagem de nutrientes em povoamentos de Pinus obliqua com 22 anos de idade, observou diferença nos teores de nutrientes, na casca e na madeira, entre a base das árvores e o ápice, e na madeira, da medula em direção à periferia do tronco (cerne - alburno).

Os resultados apresentados neste estudo são corroborados por vários outros trabalhos que verificaram variação na concentração dos nutrientes ao longo do tronco, tanto na madeira quanto na casca, entretanto, os padrões não são constantes nem para nutriente nem para espécie vegetal (ATTIWILL, 1980; ZEN; POGGIANI; COUTO, 1981; ANDRAE, 1983; ANDRAE; KRAPFENBAUER, 1983a; 1983b; FREITAS, 2000; HOPPE, 2003; RUBILAR; ALLEN; KELTING, 2005; SAIDELLES, 2005). Os padrões mais frequentes, para nutrientes com alta mobilidade como N, P e K, e de baixa mobilidade como Ca (MARSCHNER, 1995), são, respectivamente, crescente e decrescente, da base do tronco em direção ao ápice da copa (ANDRAE, 1983; ANDRAE; KRAPFENBAUER, 1983a; 1983b; HOPPE, 2003; RUBILAR; ALLEN; KELTING, 2005). Também é possível que os padrões se alterem com a idade das árvores, no entanto, Andrae (1983) e Andrae e Krapfenbauer (1983b), respectivamente, avaliaram aos 4 e 8 anos de idade a variação na concentração de nutrientes ao longo do tronco, em um povoamento de Eucalyptus saligna, e verificaram padrão constante para N, P, K, Ca e Mg, tanto na casca como na madeira.

$\mathrm{Na}$ Tabela 4 apresentam-se as equações de regressão que descrevem a concentração dos nutrientes ao longo do tronco, na casca e na madeira de Pinus taeda, aos 17 anos pós-plantio. O nível de ajuste das equações de regressão é variável entre os nutrientes em cada componente da biomassa, mas de modo geral, a casca apresentou melhor ajuste entre a concentração de nutrientes e a altura de coleta das amostras. A probabilidade de F é altamente significativa, exceto para Mn na madeira, o que em última análise atesta quão melhor são as estimativas das equações de regressão em relação à média aritmética (TM) dos teores das amostras coletadas nas diferentes posições ao longo do tronco.

A maioria das relações entre a altura de coleta das amostras e a concentração de nutrientes foi positiva, exceto para $\mathrm{Mn}, \mathrm{Ca}, \mathrm{S}$ e $\mathrm{Fe}$ na madeira e $\mathrm{Ca}, \mathrm{Zn}$ e Fe na casca. Dentre os nutrientes com relação positiva, a tendência foi linear, como para $\mathrm{Zn}$ e Fe na casca, mas principalmente exponencial, como verificado para N, $\mathrm{P}$, $\mathrm{K}, \mathrm{Mg}, \mathrm{S}, \mathrm{B}, \mathrm{Cu}$ e Mn na casca e N, P, K, Mg, B, Cu e Zn na madeira. Ainda foi possível distinguir uma relação linear negativa para Ca na madeira, ou seja, à medida que aumenta a altura de coleta de amostra a concentração de nutrientes diminui; e outra curvilinear, para $\mathrm{S}$ e Fe na madeira e Ca na casca, descrita pelas equações polinomiais, a qual apresenta os valores mais elevados para a concentração de nutrientes nas extremidades do tronco; além do padrão invariável, correlação não significativa entre a concentração de nutriente e a altura de coleta das amostras, para Mn na madeira. Com base nos diferentes graus de associação entre a altura de coleta das amostras e a concentração de nutrientes na casca e na madeira de Pinus taeda foi possível distinguir os cinco padrões para a variação dos nutrientes ao longo do tronco, apresentados na Figura 4.

Rubilar, Allen e Kelting (2005) que também estudaram a variação na concentração de nutrientes ao longo do tronco em Pinus teada, definiu padrões coincidentes com os apresentados neste trabalho, para $\mathrm{N}, \mathrm{P}, \mathrm{K}, \mathrm{Mg}, \mathrm{S}$ e $\mathrm{Mn}$ na casca e para $\mathrm{N}, \mathrm{P}, \mathrm{K}, \mathrm{Mg}, \mathrm{S}$, e $\mathrm{Mn}$ na madeira, sendo que esses autores não 
analisaram $\mathrm{Fe}$ em ambos os componentes da biomassa e $\mathrm{Cu}$ na casca. Ainda em relação a esses autores, foram verificados resultados divergentes para B na casca, com padrão invariável ao longo do tronco ao invés de exponencial; Zn na casca, com padrão exponencial ao invés de linear; e Ca na casca e na madeira, com padrões invariável e curvilinear, em vez de curvilinear e linear (negativo), respectivamente.

Considerando apenas as tendências crescente e decrescente para a concentração de nutrientes ao longo do tronco, Witschoreck e Schumacher (2013) obtiveram, para Platanus $x$ acerifolia, comportamento semelhante para N, P, K, Ca, B, Cu e Zn na madeira e N, P, K, Mg, B, Cu, Fe, Mn na casca.

Os padrões verificados neste trabalho também são comparáveis aos obtidos por Andrae e Krapfenbauer (1983a), para casca e madeira, em um estudo que avaliou a biomassa e os nutrientes: N, P, $\mathrm{K}, \mathrm{Ca}$ e Mg, em um plantio de Araucaria angustifolia com 17 anos de idade.

Tabela 4 - Parâmetros estatísticos das equações de regressão utilizadas para descrever a concentração dos nutrientes ao longo do tronco, na casca e na madeira, em um povoamento de Pinus taeda, com 17 anos de idade, em Cambará do Sul - RS.

Table 4 - Statistical parameters of regression equations used for describing nutrient concentrations in wood and bark, along the stem, in a 17-year-old Pinus taeda stand, in Cambará do Sul - RS state.

\begin{tabular}{|c|c|c|c|c|}
\hline Nutriente & Equações de regressão & $\mathbf{R}^{2}$ & Syx & Prob. $>$ F \\
\hline \multicolumn{5}{|c|}{ Madeira do tronco } \\
\hline $\mathrm{N}$ & $\ln \mathrm{y}=0,13981^{* *}+0,65297^{* *} \cdot \mathrm{h}^{2}$ & 0,593 & 0,159 & $<0,001$ \\
\hline $\mathrm{P}$ & $\ln y=-2,76474^{* *}+1,06491^{* *} \cdot h^{2}$ & 0,572 & 0,27 & $<0,001$ \\
\hline $\mathrm{K}$ & $\ln \mathrm{y}=-0,64387^{* *}+0,74759^{* *} \cdot \mathrm{h}^{3}$ & 0,522 & 0,195 & $<0,001$ \\
\hline $\mathrm{Ca}$ & $\ln \mathrm{y}=-0,28784^{* *}-0,22849^{* *} . \mathrm{h}$ & 0,146 & 0,157 & 0,001 \\
\hline $\mathrm{Mg}$ & $\ln \mathrm{y}=-1,34240^{* *}+0,34904^{* *} \cdot \mathrm{h}^{3}$ & 0,259 & 0,161 & $<0,001$ \\
\hline $\mathrm{S}$ & $\mathrm{y}=0,39095^{* *}-0,34269^{* *} \cdot \mathrm{h}+0,43353^{* *} \cdot \mathrm{h}^{2}$ & 0,226 & 0,072 & $<0,001$ \\
\hline $\mathrm{B}$ & $\ln \mathrm{y}=1,43684^{* *}+0,62574^{* *} \cdot \mathrm{h}^{3}$ & 0,406 & 0,207 & $<0,001$ \\
\hline $\mathrm{Cu}$ & $\ln y=0,24853^{* *}+1,07171^{* *} \cdot h^{3}$ & 0,655 & 0,212 & $<0,001$ \\
\hline $\mathrm{Fe}$ & $\ln \mathrm{y}=2,77055^{* *}-1,60414^{* *} . \mathrm{h}+1,82765^{* *} . \mathrm{h}^{2}$ & 0,295 & 0,216 & $<0,001$ \\
\hline $\mathrm{Mn}$ & $y=34,40$ & - & - & n.s. \\
\hline $\mathrm{Zn}$ & $\ln \mathrm{y}=1,08023^{* *}+1,33066^{* *} \cdot \mathrm{h}^{3}$ & 0,642 & 0,271 & $<0,001$ \\
\hline \multicolumn{5}{|c|}{ Casca do tronco } \\
\hline $\mathrm{N}$ & $\ln y=0,94572^{* *}+1,12481^{* *} \cdot h^{2}$ & 0,891 & 0,116 & $<0,001$ \\
\hline $\mathrm{P}$ & $\ln y=-1,51721^{* *}+1,60102^{* *} \cdot h^{2}$ & 0,796 & 0,238 & $<0,001$ \\
\hline $\mathrm{K}$ & $\ln y=-0,31261^{* *}+1,99073^{* *} \cdot h^{2}$ & 0,882 & 0,213 & $<0,001$ \\
\hline $\mathrm{Ca}$ & $y=1,75507^{* *}-3,59984^{* *} \cdot h+3,72818^{* *} . h^{2}$ & 0,37 & 0,325 & $<0,001$ \\
\hline $\mathrm{Mg}$ & $y=0,43198^{* *}+1,13025^{* *} \cdot h^{3}$ & 0,854 & 0,128 & $<0,001$ \\
\hline $\mathrm{S}$ & $\ln y=-1,04975^{* *}+0,69251^{* *} \cdot h^{2}$ & 0,746 & 0,119 & $<0,001$ \\
\hline $\mathrm{B}$ & $y=9,69646^{* *}+9,54372^{* *} \cdot h^{3}$ & 0,604 & 2,114 & $<0,001$ \\
\hline $\mathrm{Cu}$ & $y=4,44749^{* *}+1,64609^{* *} \cdot h^{3}$ & 0,292 & 0,701 & $<0,001$ \\
\hline $\mathrm{Fe}$ & $\ln \mathrm{y}=5,09607^{* *}+0,45335^{* *} . \mathrm{h}$ & 0,437 & 0,147 & $<0,001$ \\
\hline $\mathrm{Mn}$ & $\ln \mathrm{y}=3,38900^{* *}+1,00227^{* *} \cdot \mathrm{h}^{2}$ & 0,667 & 0,208 & $<0,001$ \\
\hline $\mathrm{Zn}$ & $\ln y=1,74523^{* *}+2,02175^{* *} . h$ & 0,815 & 0,276 & $<0,001$ \\
\hline
\end{tabular}

Em que: $\mathrm{y}=$ nutrientes: $\mathrm{N}, \mathrm{P}, \mathrm{K}, \mathrm{Ca}, \mathrm{Mg}$ e S em g kg-1e B, Cu, Fe, Mn e Zn em mg kg-1; h = altura relativa de coleta das amostras $(0,1 ; 0,3 ; 0,5$; $0,7$ e 0,9$)$ com base na altura total. **= significância $\mathrm{p}<0,1 ; *=$ significância $\mathrm{p}<0,5 ;$ n.s. = não significativo; $\mathrm{R}^{2}=$ coeficiente de determinação, no caso dos modelos polinomiais corresponde ao coeficiente de determinação ajustado em função do número de coeficientes; Syx = erro padrão de estimativa; Prob. > F = nível de significância para a estatística F. 
A partir das equações de regressão (Tabela 4) foi estimado o intervalo de confiança para a altura relativa de coleta de amostra, tendo como referência o TMPB, para cada nutriente e componente do tronco, além da indicação do intervalo amostral válido para todos os nutrientes na madeira (linha contínua) e na casca (linha pontilhada) (Figura 5). A estimativa mais precisa do estoque de nutriente obtida mediante a coleta e análise de várias amostras, de casca e madeira ao longo do tronco, constitui-se em um procedimento moroso e oneroso. A possibilidade de coleta de apenas uma amostra de casca e madeira por árvore-amostra apresenta grande economia de recursos. Os intervalos de confiança para a altura relativa de coleta de amostras, válidos para todos os nutrientes, foram estimados em 35 a 53\% na madeira e de 37 a 46\% para a casca. Por sua vez, o intervalo de confiança para a coleta de amostras e estimativa do estoque de todos os nutrientes de ambos os componentes foi definido pela casca, tendo como limites superior e inferior $\mathrm{N}$ $(46 \%)$ e K (37\%), respectivamente (Figura 5).

Figura 4 - Intervalo de confiança para os teores de nutrientes $(p=0,05)$, na casca e na madeira do tronco, em um povoamento de Pinus taeda, com 17 anos de idade, em Cambará do Sul - RS. (A) K na casca (padrão igual ao $\mathrm{N}, \mathrm{P}, \mathrm{Mg}, \mathrm{S}, \mathrm{B}, \mathrm{Cu}$ e Mn na casca e $\mathrm{N}, \mathrm{P}, \mathrm{K}, \mathrm{Mg}, \mathrm{B}, \mathrm{Cu}$ e $\mathrm{Zn}$ na madeira); (B) $\mathrm{S}$ na madeira (padrão igual ao Fe na madeira e Ca na casca); (C) Zn na casca (padrão igual ao Fe na casca); (D) Ca na madeira; (E) Mn na madeira.

Figure 4 - Confidence interval for nutrient contents $(\mathrm{p}=0,05)$, in stem bark and stem wood, in a 17-year-old Pinus taeda stand, in Camabará do Sul - RS state. (A) K in bark (same pattern to N, P, Mg, $\mathrm{S}, \mathrm{B}, \mathrm{Cu}$ and $\mathrm{Mn}$ in bark and $\mathrm{N}, \mathrm{P}, \mathrm{K}, \mathrm{Mg}, \mathrm{B}, \mathrm{Cu}$ and $\mathrm{Zn}$ in wood); (B) $\mathrm{S}$ in wood (same pattern to Fe in wood and $\mathrm{Ca}$ in bark); (C) $\mathrm{Zn}$ in bark (same pattern to Fe in bark); (D) $\mathrm{Ca}$ in wood; (E) $\mathrm{Mn}$ in wood.

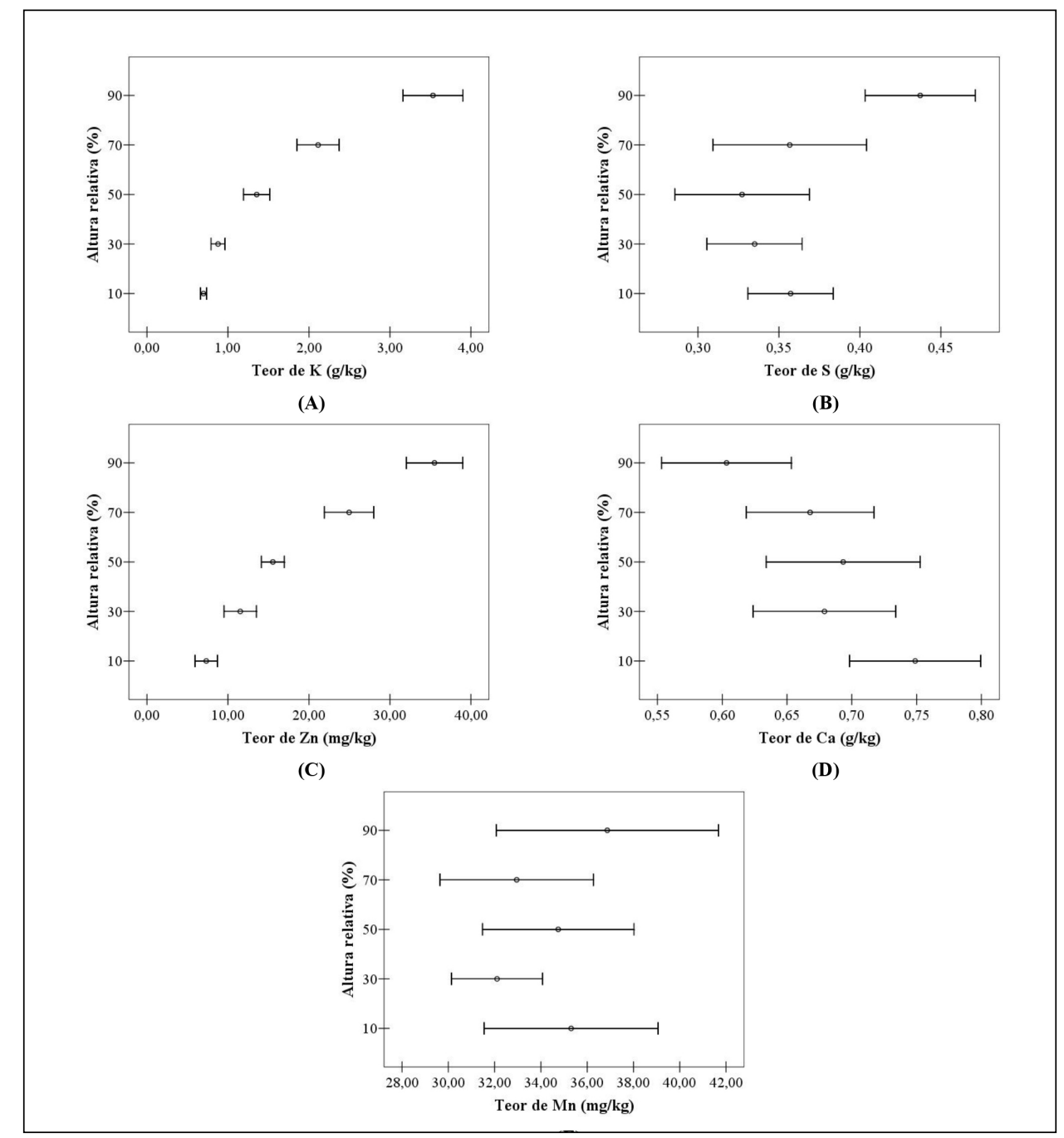


Figura 5 - Intervalo de confiança para a altura relativa de coleta de amostras de casca (.......) e madeira (-) do tronco, em um povoamento de Pinus taeda, com 17 anos de idade, em Cambará do Sul - RS.

Figure 5 - Confidence interval for relative sampling height for bark samples (…...) and stem wood samples (-), in a 17-year-old Pinus taeda stand, in Cambará do Sul - RS state.

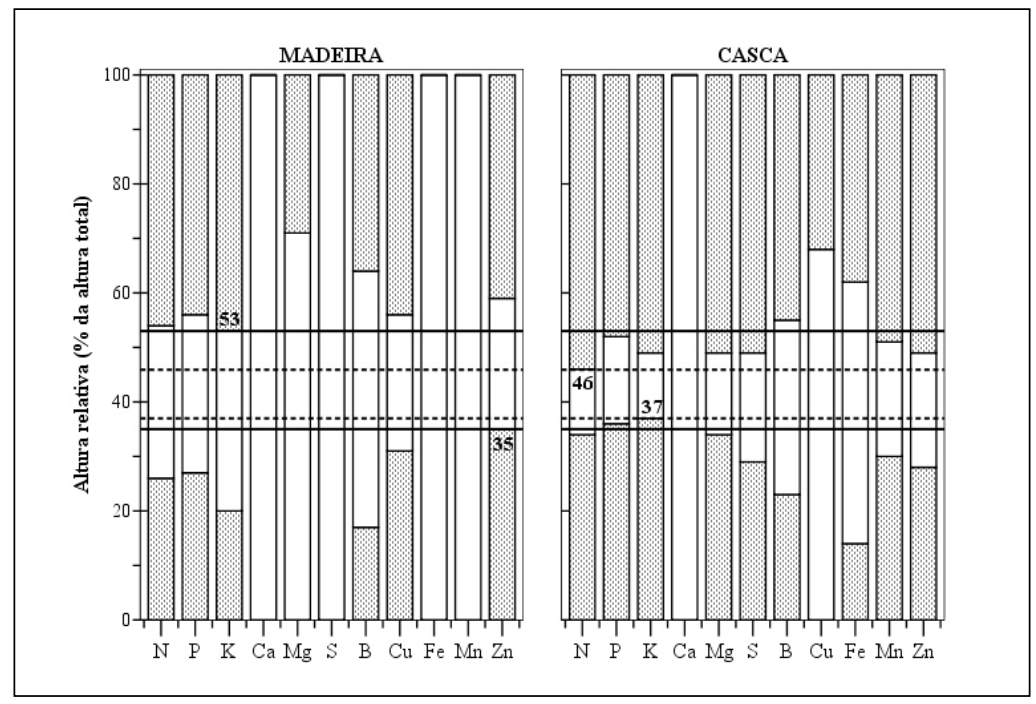

\section{Conclusões}

Existe diferença significativa na concentração da maioria dos nutrientes para casca e madeira entre amostras coletadas em diferentes posições ao longo do tronco.

A média aritmética da concentração de nutrientes (TM) em amostras coletadas em diferentes pontos ao longo do tronco não é representativa, para fins de estimativa do estoque de nutrientes, para a maioria dos nutrientes, causando, geralmente, superestimação. A ponderação da concentração em função do diâmetro (TMPD) e principalmente, em função da biomassa (TMPB) resulta em estimativas mais fidedignas.

Para as condições do estudo, a coleta de apenas uma amostra de casca e madeira entre 37 e $46 \%$ da altura total das árvores-amostra pode ser utilizada para a estimativa do estoque de nutrientes no tronco.

\section{Agradecimentos}

À Cambará S.A. pela disponibilização da área do estudo e suporte técnico, logístico e financeiro para os trabalhos de campo.

\section{Referências}

ANDRAE, F. Segundo inventário de biomassa e nutrientes de um povoamento de Eucalyptus saligna Smith no sul do Brasil. In: ANDRAE, F.; KRAPFENBAUER, A. Pesquisa austríaco-brasileira (19731982). Santa Maria, 1983. p. 86-112.

ANDRAE, F.; KRAPFENBAUER, A. Inventário de um reflorestamento de araucária de 17 anos em Passo Fundo - RS: inventário de nutrientes. In: ANDRAE, F.. Pesquisa austríaco-brasileira (1973-1982). Santa Maria: [s.n.], 1983a. p. 30-55.

ANDRAE, F.; KRAPFENBAUER, A. Estudos da situação da biomassa e nutrientes de um reflorestamento de quatro anos com Eucalyptus saligna Smith em Santa Maria - RS. In: ANDRAE, F.. Pesquisa austríacobrasileira (1973-1982). Santa Maria: [s.n.], 1983b. p. 68-85.

ATTIWILL, P. M. Nutrient cycling in a Eucalyptus obliqua (L'Hérit.) forest: IV nutrient uptake and nutrient return. Australian Journal of Botany, East Melbourne, v. 28, p. 199-222, 1980. 
BELLOTE, A. F. J.; SILVA, H. D. Sampling techniques and nutritional evaluations in eucalypt plantations. In: GONÇALVES, J. L. M.; BENEDETTI, V. (Ed.). Forest nutrition and fertilization. Piracicaba: IPEF, 2004. p. 113-139.

BURGER, L. M.; RICHTER, H. G. Anatomia da madeira. São Paulo: Nobel, 1991. 154 p.

FREITAS, R. A. Estudo da biomassa e do conteúdo de nutrientes em um povoamento de Eucalyptus grandis Hill ex Maiden plantado em solo sujeito à arenização no município de Alegrete - RS. 2000. 60 f. Dissertação (Mestrado em Engenharia Florestal) - Universidade Federal de Santa Maria, Santa Maria, 2000.

HOPPE, J. M. Biomassa e nutrientes em Platanus x acerifolia (Aiton) Willd. estabelecido no município de Dom Feliciano - RS. 2003. 143 f. Tese (Doutorado em Engenharia Florestal) Universidade de Santa Maria, Santa Maria, 2003.

INSTITUTO NACIONAL DE METEOROLOGIA (Brasil). Oitavo Distrito de Meteorologia. Seção de observação e meteorologia aplicada. Relatório $n^{\circ} 027 / 2008$, protocolado sob $n^{\circ} 4294,24 / 03 / 2008$. Porto Alegre: INMET, 2008. 4 p.

LANDSBERG, J. J. Physiological ecology of forest production. London: Academic Press, 1986. 198 p.

MARSCHNER, H. Mineral nutrition of higher plants. San Diego: Academic Press, 1995. 889 p.

MIYAZAWA, M. et al. Análises químicas de tecido vegetal. In: SILVA, F. C. (Org.). Manual de análises químicas de solos, plantas e fertilizantes. Brasília: Embrapa Comunicação para Transferência de Tecnologia, 1999. p. 171-224.

MORO, L. et al. Modelos matemáticos para estimativa de exportação de nutrientes em povoamentos de Pinus taeda L. Floresta, Curitiba, v. 37, n. 2, p. 223-230, maio/ago. 2007.

PALLARDY, S. Physiology of woody plants. San Diego: Academic Press, 2008. 454 p.

PEREIRA, A. R. et al. Concentração e distribuição de nutrientes em Eucalyptus grandis em função da idade, cultivado na região do cerrado. Brasil Florestal, Brasília, n. 59, p. 27-37, 1984.

REIS, M. G. F.; BARROS, N. F. Ciclagem de nutrientes em plantios de eucalipto. In: BARROS, N. F.; NOVAIS, R. F. (Ed.). Relação solo eucalipto. Viçosa, MG: Ed. Folha de Viçosa, 1990. p. 265-302.

RUBILAR, R. A.; ALLEN, H. L.; KELTING, D. L. Comparison of biomass and nutrient content equations for successive rotations of loblolly pine plantations on an Upper Coastal Plain Site. Biomass and Bioenergy, Oxford, v. 28, n. 6, p. 548-564, jun. 2005.

SAIDELLES, F. L. F. Determinação da biomassa e altura de amostragem para a quantificação de nutrientes em Acacia mearnsii De Wild. 2005. 97 f. Tese (Doutorado em Engenharia Florestal) Universidade Federal de Santa Maria, Santa Maria, 2005.

STRECK, E. V. et al. Solos do Rio Grande do Sul. 2. ed. Porto Alegre: EMATER/RS; ASCAR, 2008. 222 p.

WARING, R. H.; SCHLESINGER, W. H. Forest ecosystems: concepts and management. San Diego: Academic Press, 1985. 340 p.

WITSCHORECK, R. Biomassa e nutrientes no corte raso de um povoamento de Pinus taeda L. de 17 anos de idade no município de Cambará do Sul - RS. 2008. 80 f. Dissertação (Mestrado em Engenharia Florestal) - Universidade Federal de Santa Maria, Santa Maria, 2008.

WITSCHORECK, R.; SCHUMACHER, M. V. Teor e alocação de nutrientes em plantio de Platanus $x$ acerifolia (Aiton) Willd. em Dom Feliciano-RS. Ciência Florestal, Santa Maria, v. 23, n. 4, p. 667678, out./dez. 2013.

ZEN, S.; POGGIANI, F.; COUTO, H. T. Z. Variação na concentração de nutrientes ao longo do caule de Eucalyptus saligna: implicações na utilização energética dos resíduos florestais. Piracicaba: IPEF, 1981. 7 p. (Circular Técnica, 136). 